\title{
Study on Changes of the Urea, Serum Creatinine and Glomerular Protein Permeability, after General Anesthesia with Sevoflurane
}

\author{
Stoian Mircea, Stoian Adina*, Costel Dumitru, Tripon Florin, Badea ludita, Azamfirei Leonard \\ University of Medicine and Pharmacy of Tirgu Mureș, România
}

Introduction:The widespread use of sevoflurane as an induction and maintenance volatile agent of general anesthesia demostrates an increased safety profile. Sevoflurane contact with $\mathrm{CO}_{2}$ absorbents lead to the occurrence of toxic compounds such as Compund $\mathrm{A}$ and Compound B. Among the side efffects of Sevoflurane remember the renal toxic effect much discussed in the literature but still unresolved. In previous research we have demonstrated the glomerular protein changes as a result of exposure to Sevoflurane. In the current study we intend to monitor the changes in blood urea nitrogen and serum creatinine after exposure to Sevoflurane. Material and method: We included in our study 90 patients who were anesthetized in the Department of Anesthesiology of the County Mure Hospital during 01.10.2009-01.10.2014. They had normal values for blood urea nitrogen and serum creatinine and had no preoperative proteinuria. Serum and urine samples were taken preoperatively and at 24 and 72 hours postanesthetic and were analyzed in the laboratory. Proteinuria was determined by spectrophotometry. Results: After protein quantitative determination by spectrophotometry and statistical anaysis we obtained significant differences by comparing the average preoperative/24 hours total protein $(p<0.0001)$ and $24 / 72$ hours $(p<0.0001)$. There are no significant statistical differences by comparing the blood urea nitrogen at the three intervals $(p<0.53)$ and no statistical changes for mean serum creatinine $(p<0.18)$. Conclusions: Changes in glomerular filtered proteins following exposure to Sevoflurane demonstrate its toxic effect on glomerular tubules. Lack of perioperative significant wich is why we recommend determining perioperative urinary protein as a marker of glomerular damage.

Keywords: Sevoflurane, proteinuria, creatinine, blood urea nitrogen

Received: 16 March 2015 / Accepted: 19 July 2015

\section{Introduction}

On a large scale use of Sevoflurane as induction and maintaining agent of general volatile anesthesia showed an increased safety profile [1]. Sevoflurane contact with $\mathrm{CO} 2$ absorbents, leads to toxic compounds: Compound A (pentafluoroizopropenil-fluorometil- ether -PIFE) and Compound B (pentafluorometoxil-isopropyl -fluoromethylether-PME) [2]. Among the side effects of Sevoflurane, we can mention the renal toxic effect, much discussed in the literature and yet unclarified.

Experience in repeated exposure to Sevoflurane is little known, so the use of Sevoflurane in patients with renal disease, diabetes and hypertension is not restrictive. We demonstrated the changing renal permeability to proteins in other studies by geometric Electrofocuing [3]. In previous research we have shown the glomerular changes in proteins as a result of exposure to Sevoflurane [4].

In this study we aim to analyze the postanesthesia changes in the urea and serum creatinine, in relation with changes in glomerular permeability for proteins. Further on, we intend to monitor prospective urea and serum creatinine changes after exposure to sevoflurane and relate them to changes in glomerular protein. Last but not least we intend to prospectively monitor urea and serum creatinine changes after exposure to Sevoflurane and their relation to glomerular changes for proteins. In this study we present partial data on a group of 90 patients involved in this observational study. The study is prospective, observational study, which was approved by the ethics committee of the Hospital. All patients patients signed an informed consent for the collection of biological samples and their analysis.

\section{Material and method}

We have analyzed 122 patients, out of which we included in the study, considering inclusion/exclusion criteria, a total of 90 patients, who were anesthetized in the Department of Anesthesiology of the Mures County Hospital, during the period of 01.10.2009-01.11.2014.

The inclusion criteria were:

- medium (60-120 minutes) and long term (over 60 minutes) anesthesia with Sevoflurane;

- ASA I-III anesthetic risk class;

- preoperatively normal urea and creatinine serum values;

- without preoperative affection of glomerular protein.

The exclusion criteria were:

- normal preoperative serum urea and creatinine values $(0,40 \mathrm{mg} \%-1 \mathrm{mg} \%)$;

- previous kidney disease;

- anesthetic risk over ASA III,

- patients refusal to use biological samples for research;

- preoperative proteinuria. 
The anesthesia has complied with the following protocol:

- Standard monitoring was performed using ECG, non-invasive $\mathrm{TA}, \mathrm{SpO}_{2}, \mathrm{AV}$, Resp, $\mathrm{ETCO}_{2}, \mathrm{MAC}$, ET Sevo, temperature measurements.

- Anesthetic induction was performed using Midazolam, Propofol, Fentanyl and Rocuronium bromide, while for anesthesia maintenance we used Sevoflurane, Fentanyl, Rocuronium bromide.

We maintained the anestehsia with a low flow of fresh gas FGF-2 $1 /$ min at a MAC (minimum alveolar concentration) between $1,2-2 \%$ for Sevoflurane. The CO2 absorber used in the study was Soda lime, containing Sodium, Calcium hydroxide, Violet ethyl, and water. For fluid balance we used crystalloids: Ringer and $0.9 \%$ saline at a rate of administration of $500 \mathrm{ml} /$ hour and postoperative $35 \mathrm{ml} / \mathrm{kg} /$ body weight / 24 hours. Volume loss during surgery were monitored and substituted with crystalloid, colloid substances, blood and blood derivatives, so as to maintain a central venous pressure (CVP between $8-12 \mathrm{~cm}$ $\mathrm{H} 2 \mathrm{O}$ ).

We collected 122 samples of urine preoperatively, 90 urine samples 24 hours after surgery and 90 samples 72 hours after surgery, samples which were analyzed without being preserved in advance in order to avoid any conservation errors. Quantitative determination of protein in urine was performed by spectrophotometry at $600 \mathrm{~nm}$, with Konelab 30i autoanalyzer, within the specialized Laboratory of the Mures County Hospital. From the same samples we performed the electrophoretic separation of proteins in urine by geometric electrofocusing in the Department of Pathophysiology laboratory, at the UMF Tg Mures. Electrophoresis photometric evaluation was performed by scanning and computer interpretation with the Cromos programme. We collected 302 blood samples, out of which we analyzed the serum urea, the serum creatinine and the serum glucose preoperative, at 24 hours postoperatively and 72 hours postoperatively. We excluded 32 patients from the study due to changes in preanesthetic glomerular protein. We collected data such as: Age, Gender, ASA risk, BMI, Primary diagnosis, Anesthetic duration, Associated pathology (Hypertension, Diabetes, Sepsis), Anesthetic case history. The results were statistically analyzed using GraphPad Prism 6 software, we analyzed demographic data, anesthetics features, the value of $\mathrm{p}$, which we considered significantly only below 0.05 .

\section{Results}

We present the demographic data and anesthetic and surgical features in Table no. 1

After statistical analysis we obtained the following statistical significance:

- for analysis of serum creatinine at 24 hours preoperatively and 72 hours postoperatively, $\mathrm{p}=0.180$ by Anova table, $\mathrm{p}=0.1088$ by Bartlett's test - Figure 1 .

- for analysis of serum urea, at 24 preoperatively, and
Table I Demographic and perioperative data

\begin{tabular}{ccc}
\hline & & Sevoflurane \\
& $\mathrm{n}=90$ \\
\hline \multirow{2}{*}{ Age } & Average & 57.93 \\
& SD & 12.00 \\
\hline BMI & Average & 23.000 \\
Gender & SD & 3.07 \\
Anesthetic duration & M/F & $40 / 50$ \\
(min) & Average & 212.131 \\
MAC & SD & 0.25 \\
\hline Anesthetic case history & & $1.2(0.9-1.3)$ \\
Diabetes & & $52.5 \%$ \\
\hline ASA & I & $14.8 \%$ \\
\hline Sepsis & II & $1.6 \%$ \\
\hline Surgery & III & $39.3 \%$ \\
Pulmonary & & $60.7 \%$ \\
General & & $21.3 \%$ \\
\hline
\end{tabular}

\section{One-way ANOVA data}

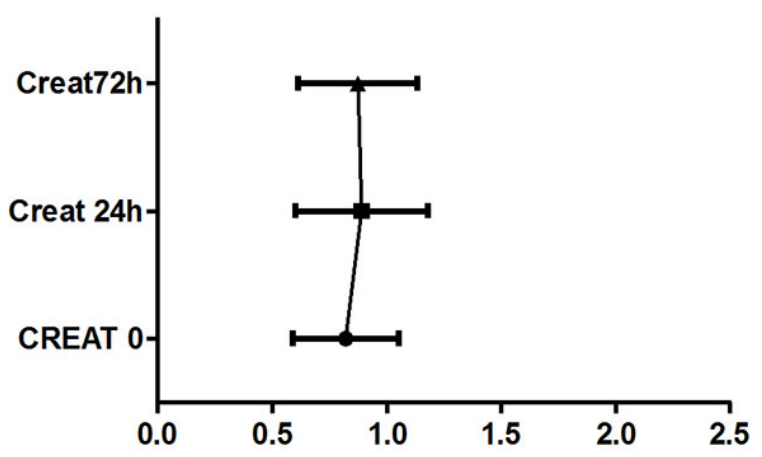

Fig. 1. Serum creatinine (mg\%)

\section{One-way ANOVA data}

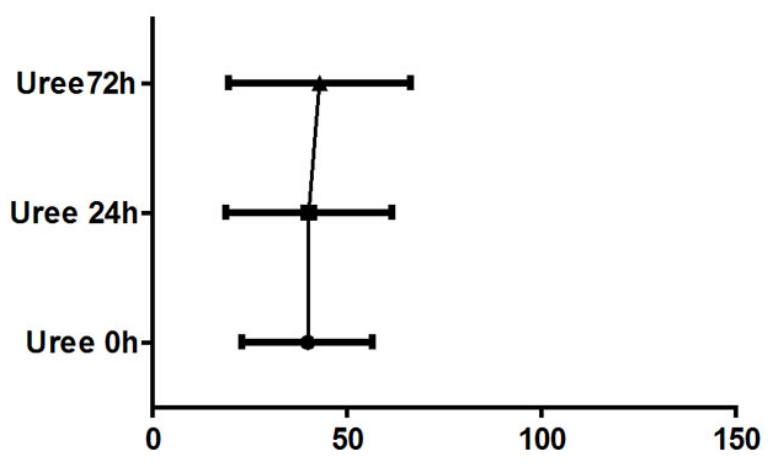

Fig. 2. Serum urea (mg\%)

72 hours postoperatively, $\mathrm{p}=0.5307$ by Anova table, $\mathrm{p}=0.006$ by Bartlett's test - Figure 2

- for analysis of preanesthetic blood glucose, and postanesthetic at 24 and 72 hours, $\mathrm{p}=0.0012$ through Anova table and $\mathrm{p}=0.001$ by Bartlett's test - Figure 3 . - for total proteins/24 hours in urine samples at the 3 


\section{One-way ANOVA data}

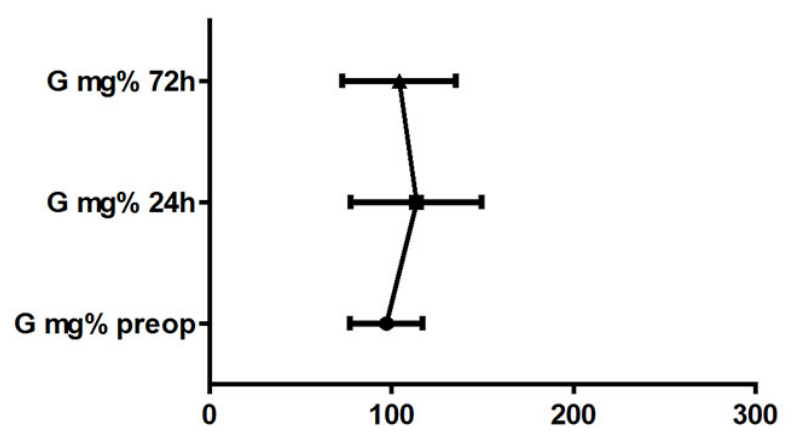

Fig. 3. Serum glucose $(\mathrm{mg} \%)$

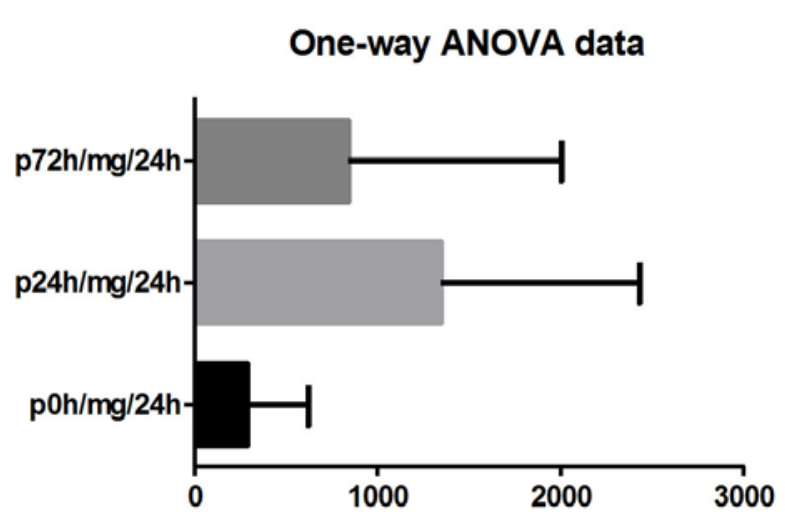

Fig. 4. Urinary proteins (mg\%)

time frames: preanesthetic, postanesthetic at 24 and 72 hours, $\mathrm{p}<0.0001$ by Anova table, $\mathrm{p}<0.0001$ by Bartlett's test -Figure 4.

\section{Discussions:}

Sevoflurane is a modern inhalation anesthetic, first introduced in 1990 in Japan by Pharmaceuticals Maruischi and starting with 1995 is marketed by Abbott Laboratories under the name of Ultiva and Sevorane [5]. After 2006 there was a new formula of sevoflurane, marketed by Baxter Healthcare which is considered therapeutically equivalent, but with important differences. In 2007, the European Medicines and Products Regulatory Agency recommends vigilance in using Penelon Sigma Delta vapourisers and withdrawn it from use, due to the interaction of sevoflurane with Lewis acids [6,7]. Currently there are 3 forms of sevoflurane, with differences in terms of the production process, but without physical-chemical differences [8] There are authors who support the use of sevoflurane in patients with renal disorder, renal transplantation [9], in anesthesia for interventions of kidney transplantation [10].

FDA warns that taking more than $2 \mathrm{MAC}$ sevoflurane concentrations and FGF (Fresh gas flow) between 1 and 2 $\mathrm{L} / \mathrm{min}$ may be associated with proteinuria and glycosuria
[11] The results of several studies are recommendations to avoid the use of high concentrations of Sevoflurane, over 1.5 MAC, at low flows of fresh gas (FGF) under $21 / \mathrm{min}$. The presence of high concentrations of protein or albumin in the urine increases the risk of progression of renal disease, increases cardiovascular morbidity, risk that is linear and continuous [12].

The changes of the glomerular filtered proteins following exposure to Sevoflurane demonstrate toxic effect on the glomerular tubules, without changes in the urea and in the serum creatinine [13]. It is to be mentioned that all patients in the study were carefully monitored hemodynamically, maintaining the CVP limits between $8-12 \mathrm{~cm} \mathrm{H} 2 \mathrm{O}$, while keeping the values of TAM between $60-80 \mathrm{mmHg}$. It was not prescribed drugs with known nephrotoxic effect such as aminoglycosides or Vancomycin.

Statistically analyzing the partial data collected from patients involved in the study, we observed changes in the excreted proteins with a p-value $<0.0001$ and significant glycosuria $\mathrm{p}<0.0001$. The serum creatinine changes are not significant in the applied tests $\mathrm{p}=0.18$, we consider insignificant changes urea with $\mathrm{p}=0.5307$.

Noteworthy is the large number of patients with proteinuria, among those with no case history of renal disease, which led to the exclusion of 32 patients from the group.

\section{Conclusions}

Following partial data analysis of this study, we observed significant presence of protein and carbohydrates in postoperatively urine, with a peak at 24 hours postanesthetic and regression trend towards 72 hours, without return to initial, pre-anesthetic values.

Statistically insignificant urea and creatinine values demonstrate their perioperative irrelevance and therefore we recommend the determination of other markers of kidney disease, such as urinary proteins, albuminuria, due to simple determining and low cost. The routine use of sevoflurane widely, without taking into account the possible effect of renal toxicity in patients with diabetes, hypertension, sepsis is dangerous, which is why we recommend rigorous monitoring of renal function or use other techniques such as TIVA with anesthetics Propofol.

\section{Acknowledgement}

This paper was published under the frame of European Social Found, Human Resources Development Operational Programme 2007-2013, project no. POSTDRU/1 $59 / 1.5 / S / 136893$

\section{References}

1. Fukazawa K, Lee HT. Volatile anesthetics and AKI: risk, mechanisms, and a potential therapeutic window. J Am Soc Nephrol, 2014;25:884892.

2. Lee HC, Kim D, Ahn W, Sim J, Chung Y. Comparison of the renal safety between carbon dioxide absorbent products under sevoflurane anesthesia: a pilot study. Korean J Anesthesiol, 2012;63:11-17.

3. Stoian M, Stoian Adina, Cozma D, et al. Modification of Renal Permeability for Proteins after General Anesthesia with Sevoflurane and 
Desfluran. Acta Medica Marisiensis, 2011;57 (suppl 3):23-24.

4. Stoian M, Stoian A, Onisor D, Brusnic O, Cozma D, Buicu F. Urine protein separation by geometrical electrophoresis after anaesthesia with sevoflurane. Acta Medica Transilvanica, 2012;2:159-162.

5. BakerbMT. Sevoflurane: are there differences in product? Anesth Analg, 2007; 1447-51.

6. ONeil B, Hafiz MA, DeBeert DA. Corosion of Penelon Sevoflurane Vaporizer. Anaesthesia, 2007;62:421.

7. Gupta A, ElyJ. Faulty sevoflurane vaporizer. Anaesthesia, 2007;62:421

8. Alfredo AVP, Solange ML, Deise MR, Maria IMR, Santiago OQ. A doubleblind comparative study between generic Sevoflurane and Sevorane. Rev Bras Anesthesiolol, 2010;60:Campinas Sept./oct
9. A Ergolu, E Erturk, $\mathrm{H}$ Bostan. Sevofluran Anaesthesia in a patient with renal transplantation: Case Report and literature Reviw. The Journal of anesthesiology, 2006;13:1.

10. Jin Ha Park, Jae Hoon Leel, Dong Jin Joo, Ki Jun Song, Yu Seun Kim, and Bon-Nyeo Koo. Effect of sevoflurane on grafted kidney function in renal transplantation. Korean J Anesthesiol, 2012;62:529-535.

11. FDA Professional Drug Information:

12. RM Bermudez, SG Garcia, DP Surribas, et. al. Consensus Document. Recommandations on assessing proteinuria during the diagnosis and follow-up of cronic kidney disease. Nefrologia 2011;31:331-345.

13. Hines RL, Marschall KE. Stoeltings's Anesthesia and co-existing disease. Renal Tubular Function and Integrity, 2012;Chapter 17 Renal Disease:336-337. 